\title{
Uso de estrategias metacomprensivas para el fortalecimiento de la comprensión lectora en estudiantes de segundo ciclo de un colegio oficial en Bogotá, Colombia ${ }^{1}$
}

\author{
Use of Meta-comprehensive Strategies to Strengthen Reading \\ Comprehension in Second-Cycle Students from a Public School at \\ Bogotá, Colombia
}

\author{
Uso de estrategias metacomprênsivas para o fortalecimento da \\ compreensão leitora em estudantes de segundo ciclo de uma escola \\ oficial em Bogotá, Colômbia
}

\author{
Bibian Alexandra Rodríguez Ribero ${ }^{2}$ \\ María Eugenia Calderón Sánchez ${ }^{3}$ \\ Martha Helena Leal Reyes ${ }^{4}$ \\ Nicolás Arias-Velandia ${ }^{5}$
}

Resumen

Esta investigación indagó el efecto de una intervención en aula usando la metacomprensión con énfasis en la formulación de autopreguntas, en la mejora de la comprensión lectora de estudiantes de tercero y cuarto grados de primaria. La comprensión lectora se entendió como proceso que relaciona contenido, texto e inferencias, facilitada por la metacomprensión, o uso de actividad metacognitiva en el trabajo de comprensión lectora. Participaron dos grupos de estudiantes de tercero y cuarto grados: el grupo de intervención (que tuvo una evaluación inicial, un trabajo de aula en metacomprensión formulando autopreguntas -implementado por las tres primeras autoras de este artículo-y una evaluación final) y el grupo de comparación (que tuvo una evaluación inicial, el trabajo de aula que venían siguiendo habitualmente con sus docentes y una evaluación final). Se aplicó la prueba de comprensión lectora Comprensión Lingüística Progresiva (CLP, Alliende, Condemarín \& Milicic, 2004) y el cuestionario sobre estrategias metacomprensivas en la evaluación inicial y final con ambos grupos. Dicha información se complementó con datos de entrevistas (aplicadas una sola vez) a otros docentes (que no eran los investigadores) que también laboran en la institución educativa, con el fin de conocer la perspectiva que ellos tenían sobre comprensión lectora, que pudiese respaldar sus prácticas de enseñanza de la lectura. Los resultados mostraron aumento en las puntuaciones en comprensión lectora y diversificación de las estrategias metacomprensivas solamente en el grupo de intervención, y diferentes concepciones de los demás docentes sobre la comprensión lectora, que no relacionaron con estrategias metacomprensivas.

Palabras clave: Lectura, comprensión lectora, metacognición, estrategias metacomprensivas, autopreguntas

1 Artículo derivado de la tesis de maestría desarrollada por las tres primeras autoras bajo la dirección de Nicolás Arias-Velandia, para optar al título de magíster en Pedagogía, Facultad de Educación, Universidad de La Sabana, Chía, Colombia. Las tres primeras autoras desarrollaron este trabajo durante dichos estudios de maestría bajo el auspicio del programa "Maestros Empoderados con Bienestar y Mejor Formación" de la Secretaría de Educación del Distrito, Bogotá.

2 Colegio Alfredo Iriarte, institución oficial de educación inicial, básica y media adscrita a la Secretaría de Educación del Distrito (sED), Bogotá. Maestría en Pedagogía, Universidad de La Sabana, Chía, Colombia. Correo electrónico: bibianrori@ unisabana.edu.co

3 Colegio Alfredo Iriarte, institución oficial de educación inicial, básica y media adscrita a la Secretaría de Educación del Distrito (SED), Bogotá. Maestría en Pedagogía, Universidad de La Sabana, Chía, Colombia. Correo electrónico: mariacalsa@ unisabana.edu.co

4 Colegio Alfredo Iriarte, institución oficial de educación inicial, básica y media adscrita a la Secretaría de Educación del Distrito (sED), Bogotá, Colombia. Maestría en Pedagogía, Universidad de La Sabana, Chía, Colombia. Correo electrónico: marthalere@unisabana.edu.co

5 Maestría en Pedagogía, Facultad de Educación, Universidad de La Sabana. Autor correspondiente. Correo electrónico: nariasv@gmail.com 
This research inquired about the effect of a classroom intervention in metacomprehension strategies, specifically self-questioning, on reading comprehension improvement in third and fourth - graders. Reading comprehension was understood as a process that relates content, text and inferences, and is facilitated by metacomprehension or metacognitive processes applied to reading comprehension. The participants were third- and fourth-grade students: the intervention group (which had an initial assessment, and were submitted to classroom intervention in metacomprehension using self-questioning -implemented by the first three authors-, and had a final assessment), and the control group (which had initial assessment, the usual classroom work with other teachers, and final assessment). The Comprensión Lingüística Progresiva (CLP, Alliende, Condemarín \& Milicic, 2004)—a reading comprehension test-and the students' metacomprehensive strategies questionnaire were administered to students in both groups, in the initial and final assessments. This information was complemented with interview data from other teachers (different from the authors) in order to know their perspective on reading comprehension underlying their reading teaching practices. Results showed a rise in reading comprehension tests scores and diversification in the use of metacomprehensive strategies only in the intervention group, and different conceptions of reading comprehension in other teachers, which had nothing to do with metacomprehension strategies.

Keywords: Reading, reading comprehension, metacognition, metacomprehension strategies, self-questioning

Resumo

Esta pesquisa investigou o efeito de uma intervenção na sala de aula usando metacompreensão com ênfase na formulação de autopreguntas, na melhoria da compreensão da leitura dos estudantes nas classes de terceiro e quarto da escola primária. A Compreensão de leitura foi entendida como um processo relacionado com conteúdo, texto e inferências, facilitada pela metacompreensão, ou o uso de atividade metacognitiva no trabalho de compreensão de leitura. Participaram dois grupos de alunos de terceiro e quarto grado: o grupo de intervenção (que teve uma avaliação inicial, um trabalho de metacompeensão em sala de aula formulando autoperguntas -implementada pelas três primeiras autoras deste artigo — e uma avaliação final) e o grupo de comparação (que teve uma avaliação inicial, o trabalho em sala de aula que geralmente vem vindo junto com seus professores e uma avaliação final). Se aplicou o teste de compreensão de leitura Compreensão de Linguagem Progressiva (CLP, Alliende, Condemarín \& Milicic, 2004) e 0 Questionário sobre estratégias metacompreensivas na avaliação inicial e final com ambos grupos. Esta informação foi complementada com dados de entrevistas (aplicadas uma vez) a outros professores (que não eram investigadores) que também trabalham na escola, a fim de conhecer a perspectiva que tinham da compreensão de leitura, o que poderia sustentar suas práticas de ensino da leitura. Os resultados mostraram aumento na pontuação na leitura e diversificação de estratégias metacompreensivas apenas no grupo de intervenção, e diferentes concepções de outros professores sobre a compreensão da leitura, que não relacionaram com estratégias metacompreensivas.

Palavras Chave: Leitura, Compreensão leitora, Metacognição, Estratégias metacompreensivas, Auto pergunta

Artículo recibido el 23 de julio de 2015 y aprobado el 27 de enero de 2016

\section{Introducción}

La comprensión lectora es un tema frecuente de investigación. Prueba de ello es que sea parte de los aspectos evaluados típicamente en el logro educativo de estudiantes en pruebas censales o que aplican los Estados a sus estudiantes, para indagar acerca de la calidad de la formación que imparten las instituciones educativas (Sistema Nacional de Evaluación de la Educación, Icfes, 2013). Este trabajo busca examinar los efectos de una estrategia de trabajo en comprensión de lectura desde la perspectiva de la metacomprensión. Por este motivo, esta introducción aborda tres partes: marco conceptual de la comprensión lectora, antecedentes de investigación en comprensión lectora y resultados de la Institución Educativa Alfredo Iriarte (objeto de este estudio) en las pruebas estatales que dan cuenta del desempeño de sus estudiantes en comprensión lectora.

\section{Comprensión lectora, metacognición y metacomprensión}

La lectura es un proceso complejo de interacción entre lector y texto, mediados por el contexto. En ella se hallan implícitos procesos de orden perceptual, lingüístico y cognitivo, que permiten construir significado con el propósito de llegar a comprender lo leído (Flórez-Romero, Restrepo \& Schwanenflugel, 2007; Dubois, 2000). La comprensión lectora es un proceso de interacción entre lector, texto y contexto, que posibilita al lector construir significados a partir del uso de acciones lingüísticas, de conocimientos previos y de la realización de inferencias con el fin de interpretar y de darle sentido al texto (Cassany, 2006; Flórez-Romero et al., 2007; Smith, 1985).

De acuerdo con Flórez-Romero, Arias-Velandia y Torrado (2006) y con Scarbourough (2002), la comprensión es el aspecto de la lectura que se 
relaciona con pensar estratégicamente. Se domina en la medida en que se acerca más a las inferencias (Cuetos Rodríguez \& Ruano, 2004; Gil-Chaves \& Flórez-Romero, 2011; Graesser, Singer \& Trabasso, 1994; Orozco, 2003), a la explicitación de propósitos y relaciones que plantea el texto como unidad significativa (Perfetti, 1999), y lleva a formularse preguntas sobre su sentido y las relaciones entre sus componentes (Arias-Velandia \& Torrado, 2006; Flórez-Romero, Mateus, Santiago, Castillo \& Rodríguez, 2012). Por tal motivo, la comprensión de lectura se ha relacionado frecuentemente con la metacognición (Flórez-Romero, Torrado, ArévaloRodríguez, Mesa, Mondragón \& Pérez, 2005; Mayor, Suengas \& González, 1995).

La metacognición es el control sobre los propios procesos de pensamiento mediante su autorregulación (Burón, 1999; Flavell, 1976). Para Flavell, Baker y Brown (1984) la metacognición tiene tres etapas: la planificación, el monitoreo durante el proceso y la evaluación o constatación del conocimiento alcanzados. La metacomprensión es un subproceso de la metacognición que hace referencia a la habilidad que tiene el lector para controlar las acciones cognitivas que se desarrollan en la comprensión lectora (Burón, 1999) mediante los procesos de planeación, verificación y evaluación. Estos tres momentos se traducen en tres fases: (a) Habilidades de planificación o preparación para la lectura: ideas previas, motivación (objetivos lectores) y decisión sobre qué técnicas (herramientas) comprensivas se utilizarán; (b) Habilidades de supervisión o aplicación efectiva de las técnicas mientras se lee para darse cuenta de si se produce comprensión o no, y (c) Habilidades de evaluación o de determinación, una vez terminada la lectura, de todo aquello que ha sido útil para comprenderla.

Schmitt y Baumann (1989) y Condori (2003) exponen las siguientes estrategias de trabajo con la metacomprensión: predicción y verificación; revisión a vuelo de pájaro; autopreguntas; uso de conocimientos previos; $y$ aplicación de estrategias definidas, como el resumen, los mapas conceptuales, los cuadros comparativos, el uso de diccionario, entre otras. Diferentes estudios que han examinado la comprensión lectora ofrecen relaciones causales de análisis y estrategias para fomentar su adecuado desarrollo, desde la metacognición y su subproceso aplicado a la lectura, hasta la metacomprensión, como categorías de análisis. Dichos antecedentes de investigación se exponen a continuación.

\section{Antecedentes de investigación en comprensión lectora}

La mayoría de estos estudios coinciden en concluir que al aplicar estrategias meta-comprensivas en la práctica de la lectura de textos fortalece el desarrollo del proceso comprensivo lector y la potenciación de las habilidades metacomprensivas, lo cual lleva a un mejor desempeño escolar. En el estudio correlacional de Flórez-Romero et al. (2005) sobre las habilidades metalingüísticas, operaciones metacognitivas y su relación con las competencias en lectura y escritura en niños de primero a cuarto grados de primaria pertenecientes a tres colegios de Bogotá, Colombia, se encontró que, a medida que los estudiantes poseen mayor control y conocimiento de los procesos metacognitivos, su desempeño en lectura y escritura mejora.

Un primer conjunto de estudios muestra que la aplicación de estrategias metacomprensivas parece mejorar las capacidades de comprensión lectora de los estudiantes. Adrianzén (2012) realizó un estudio sobre la incidencia de las habilidades metacognitivas en la comprensión lectora, para lo cual aplicó un programa de habilidades metacognitivas en este aspecto con niños de cuarto grado de primaria del Colegio Parroquial Santísima Cruz de Chulucanas, de Piura, Perú, el cual mejoró la comprensión lectora de los estudiantes. Heit (2012) obtuvo resultados similares al examinar la eficacia de estrategias metacognitivas de comprensión lectora en la asignatura de Lengua y Literatura. Igual efecto encontraron Quaas, Ascorra y Bertoglia (2005) cuando indagaron la relación entre los niveles de rendimiento de los estudiantes de octavo grado de Valparaíso, Chile, en la asignatura de Lenguaje y Comunicación y las estrategias metacomprensivas que usan en la lectura. 
Universidad Pedagógica Nacional

Facultad de Humanidades

Lo mismo ocurrió en el estudio de Santibáñez y Pisconte (2003), cuando buscó probar la efectividad de un programa de reforzamiento de lectura basado en el desarrollo de habilidades metalectoras en educandosde segundo ciclo deestudios generales en la Universidad Nacional de Piura, en el Perú.

Otra investigación quiso establecer la relación entre estrategias metacomprensivas en lectura y otros factores que pueden incidir en el logro académico de los estudiantes. Wong y Matalinares (2011) realizaron un estudio para establecer la relación entre las estrategias de metacomprensión lectora y los estilos de aprendizaje de los estudiantes en Lima, Perú. Encontraron que todos los estudiantes presentan un bajo nivel en el uso de estrategias metacomprensivas con independencia de su estilo.

Un tercer conjunto de investigaciones indagó la relación entre comprensión lectora y la aplicación de algunas estrategias metacomprensivas específicas. Irrazabal, Saux, Burin y León (2006) y Nieto (2005) hallaron que realizar resúmenes funciona como estrategia metacomprensiva que permite el fortalecimiento de la comprensión lectora, y Nieto (2005) mostró además sus efectos sobre habilidades de metacomprensión. En ambos estudios participaron 281 niños, a quienes se les administró la Escala de Conciencia Metacognitiva de Lectura (ECML) y una prueba de comprensión lectora en un diseño experimental pretest-postest con grupo de comparación. En la misma línea, Condori (2003) llevó a cabo un estudio experimental que buscaba mejorar la comprensión lectora de los estudiantes haciendo uso de las estrategias metacomprensivas de predicción y verificación, revisión a vuelo de pájaro, establecimiento de propósitos y objetivos, autopreguntas, uso de conocimientos previos, resúmenes y aplicaciones de estrategias definidas.

\section{Resultados de la Institución Educativa Alfredo Iriarte en las pruebas estatales que dan cuenta del desempeño de sus estudiantes en comprensión lectora}

Desde otro punto de vista, también se han propuesto análisis de la comprensión lectora que han tenido como fin identificar y potenciar los diferentes niveles de comprensión en los educandos, para de esta manera fomentar el proceso comprensivo lector $y$ obtener un mejor desarrollo de sus competencias comunicativas (Ministerio de Educación Nacional, 2006; Icfes, 2012). Así, otra arista para plantear la presente investigación es el reporte de las pruebas Saber del año 2012 de la IED Alfredo Iriarte en el área de lenguaje, que se ilustran en el cuadro 1 y la tabla 1.

Cuadro 1. Nivel que alcanzan los estudiantes de tercero y quinto grados de la IED Alfredo Iriarte en las pruebas Saber del año 2012 en el área de lenguaje

\begin{tabular}{|l|c|c|}
\hline \multicolumn{1}{|c|}{ Nivel de los estudiantes } & $\begin{array}{c}\text { Tercer } \\
\text { grado }\end{array}$ & $\begin{array}{c}\text { Quinto } \\
\text { grado }\end{array}$ \\
\hline Insuficiente & $7 \%$ & $7 \%$ \\
\hline Mínimo & $28 \%$ & $42 \%$ \\
\hline Satisfactorio & $43 \%$ & $41 \%$ \\
\hline Avanzado & $23 \%$ & $11 \%$ \\
\hline
\end{tabular}

Fuente: Elaboración propia con base en Icfes (2013).

Tabla 1. Resultados de competencias y componentes en el área de lenguaje de los estudiantes de tercero y quinto grados de la ied Alfredo Iriarte en las pruebas Saber del año 2012

\begin{tabular}{l|c|c|} 
& $\begin{array}{c}\text { TERCER } \\
\text { GRADO }\end{array}$ & $\begin{array}{c}\text { CUARTO } \\
\text { GRADO }\end{array}$ \\
\hline Competencia comunicativa lectora & Débil & Débil \\
\hline Competencia comunicativa escritora & Débil & Débil \\
\hline Componente semántico & Fuerte & Débil \\
\hline Componente sintáctico & Débil & Fuerte \\
\hline Componente pragmático & Débil & Débil \\
\hline
\end{tabular}

Fuente: Elaboración propia con base en Icfes (2013).

En tercer grado, el 33\% de los estudiantes se encuentran ubicados en niveles insuficiente y mínimo, y el 66\% en los niveles satisfactorio y avanzado. Por otra parte, de los estudiantes en quinto grado el $49 \%$ se ubicaron en niveles insuficiente y mínimo, y el 52\% en niveles satisfactorio y avanzado (véase el cuadro 1). Esto suscita interrogantes por el alto porcentaje de estudiantes en los niveles más bajos de desempeño (insuficiente y mínimo) y sobre el aumento de estudiantes en estos niveles al pasar de tercero a quinto grado.

Con respecto a las competencias en la prueba, se reporta debilidad en la competencia comunicativa lectora y en la competencia comunicativa escrita 
(véase el cuadro 2). En cuanto a los componentes de la prueba en el área de lenguaje, los estudiantes de quinto grado presentan dificultades en el componente semántico, lo que no ocurre con tercer grado. Por el contrario, los de tercer grado presentan dificultades en el componente sintáctico, lo que no ocurre con los de quinto grado. Sin embargo, los alumnos de ambos grados presentan dificultades en el componente pragmático (véase el cuadro 2). Dicho componente está altamente relacionado con la comprensión lectora, ya que aborda el para qué se dice algo, por qué, cuándo y quién lo dice, qué podría suceder, las conjeturas o deducciones, etc., en función de una situación comunicativa (FlorézRomero, Restrepo \& Schwanenflugel, 2007).

\section{Pregunta de investigación}

Los resultados de los estudiantes de básica primaria del Colegio Alfredo Iriarte en las pruebas de Estado han mostrado bajos niveles en sus competencias asociadas a la comprensión lectora. Adicionalmente, los antecedentes de investigación muestran un efecto positivo de las estrategias metacomprensivas, y metacognitivas en general, en la comprensión lectora de estudiantes en diferentes investigaciones realizadas en España y América Latina (Adrianzén, 2012; Heit, 2012; Quaas, Ascorra \& Bertogia, 2005; Santibáñez \& Pisconte, 2003; Wong \& Matalinares, 2011). Incluso algunos de estos estudios han avanzado al punto de establecer este efecto positivo en relación con algunas estrategias metacomprensivas específicas, como la elaboración de resúmenes, la predicción y la verificación, la revisión a vuelo de pájaro, el establecimiento de propósitos y objetivos, las autopreguntas y el uso de conocimientos previos (Condori, 2003; Irrazabal etal., 2006; Nieto, 2005).

La investigación de Silvestri (2006) coincide en la idea de formular preguntas como una estrategia de metacomprensión que puede influenciar positivamente la comprensión lectora de los estudiantes. En este estudio se pretende avanzar un poco más en este camino e indagar acerca del efecto de las autopreguntas como estrategia metacomprensiva sobre el proceso comprensivo lector (Blythe \& Perkins, 1999; Schmitt \& Bauman, 1989).

Por lo tanto, esta investigación parte de la siguiente pregunta: ¿De qué manera la implementación de estrategias metacomprensivas con énfasis en el uso de autopreguntas contribuye a la mejora en la comprensión lectora de los estudiantes de tercer y cuarto grados del Colegio Alfredo Iriarte?

Con base en esta pregunta y en los antecedentes citados, se plantea la hipótesis de que la adquisición de estrategias metacognitivas con énfasis en el uso de autopreguntas contribuye a mejorar la comprensión lectora de los estudiantes de tercer y cuarto grados del Colegio Alfredo Iriarte.

\section{Método}

\section{Tipo de estudio y diseño}

Este estudio es una investigación de orientación empírico-analítica de alcance explicativo (Hernández-Sampieri, Fernández Collado \& Baptista Lucio, 2010), que utiliza técnicas de análisis cuantitativas y cualitativas (Onwuegbuzie \& Leech, 2006). Utiliza un diseño cuasi-experimental, porque maneja evaluaciones previas y posteriores a un programa implementado y tiene un grupo de intervención (que trabaja con la estrategias en aula de metacomprensión implementadas por las tres primeras investigadoras autoras en este reporte) y un grupo de comparación (que sirve de control, en el cual otros docentes de la institución realizan la instrucción que habitualmente siguen en la enseñanza del español como primera lengua). Ambos grupos están conformados por estudiantes en los mismos grados y con características similares, que no fueron elegidos al azar (están dados por los cursos previamente establecidos en la institución educativa) y no tuvieron todas las condiciones de control ambiental por trabajar con ellos en su contexto natural de aula (Hernández-Sampieri et al., 2010).

Las variables abordadas por este estudio se presentan en la tabla 2 . 
Tabla 2. Variables del estudio

\begin{tabular}{|c|c|c|}
\hline TIPO DE VARIABLE(S) & VARIABLE & INDICADORES Y EVIDENCIAS \\
\hline $\begin{array}{l}\text { Independiente } \\
\text { (Trabajo controlado por los investigadores } \\
\text { que supone efectos en las variables de- } \\
\text { pendientes) }\end{array}$ & $\begin{array}{l}\text { Trabajo de docentes investigadoras en es- } \\
\text { trategias metacomprensivas }\end{array}$ & $\begin{array}{l}\text { Plan de intervención basado en estrategias } \\
\text { metacomprensivas mediante autopregun- } \\
\text { tas Meprecom }\end{array}$ \\
\hline \multirow{2}{*}{$\begin{array}{l}\text { Dependientes o efectos } \\
\text { (Efectos que se busca producir por el tra- } \\
\text { bajo con la variable independiente) }\end{array}$} & \multirow{2}{*}{$\begin{array}{l}\text { Avance en comprensión lectora de los es- } \\
\text { tudiantes } \\
\text { Cambios en estrategias metacomprensi- } \\
\text { vas manifestadas por los estudiantes }\end{array}$} & $\begin{array}{l}\text { Prueba CLP } \\
\text { Registros de observación }\end{array}$ \\
\hline & & Cuestionario a estudiantes \\
\hline $\begin{array}{l}\text { Asociada } \\
\text { (Aspecto que puede influir en las variables } \\
\text { dependientes que se caracteriza pero que } \\
\text { los investigadores no controlan) }\end{array}$ & $\begin{array}{l}\text { Ideas manifiestas de los docentes sobre la } \\
\text { comprensión lectora }\end{array}$ & Entrevista a otros docentes de la institución \\
\hline
\end{tabular}

\section{Participantes}

Ochenta y tres (83) estudiantes y ocho (8) docentes del ciclo dos, tercer y cuarto grado, de la Institución Educativa Distrital Alfredo Iriarte de Bogotá, Colombia, entidad educativa oficial adscrita a la Secretaría de Educación del Distrito de la misma ciudad. Los estudiantes se encontraban en edades entre 7 y 10 años. La mayoría de ellos habitaban en núcleos familiares monoparentales de estrato uno ${ }^{6}$ y se encontraban cursando por primera vez tercer y cuarto grados de Educación Básica Primaria. Cuarenta y nueve (49) de los estudiantes del ciclo dos de la sede $\mathrm{C}$ formaron parte del grupo de intervención. Los restantes treinta y cuatro (34) estudiantes de la sede B jornada mañana conformaron el grupo de comparación.

\section{Instrumentos y técnicas de}

recolección de información

\section{Prueba de Comprensión Lingüistica Progresiva (CLP)}

Esta prueba estandarizada y adaptada para población estudiantil fue diseñada por Alliende, Condemarín $\&$ Milicić (2004). Es un instrumento que permite medir el grado de dominio de la lectura convencional por parte del niño desde el aprendizaje inicial

6 Información tomada de las hojas de matrícula de los estudiantes para los años 2013, 2014 y 2015. hasta el momento en que se convierte en un lector independiente.

Se aplicó el test 3 de la prueba cLP debido a que engloba características de desarrollo comprensivo lector que se persiguen en Colombia de conformidad con los Estándares básicos de competencias del Lenguaje para el ciclo dos (Ministerio de Educación Nacional, 2006). En la sede C, grupo de intervención, se analizaron 49 pruebas del total de sujetos que participaron en la aplicación previa y posterior a la intervención realizada ( 26 en tercer grado y 23 en cuarto). En la Sede B, grupo de comparación, se analizaron 34 pruebas, de 14 estudiantes de tercer grado y 20 de cuarto, realizadas también antes y después de la intervención desarrollada en el otro grupo.

\section{Entrevista a otros docentes de la institución}

Esta técnica de recolección de información fue un cuestionario con el que se indagó acerca de tres aspectos en todos los docentes de segundo ciclo de los estudiantes de ambas sedes, que no participaron como investigadores y que siguieron realizando sus prácticas habituales de enseñanza de la comprensión lectora con estudiantes de primaria: sus conceptos sobre comprensión lectora, las estrategias que declaran usar en el aula para el desarrollo de la comprensión lectora y las principales causas a las que atribuyen la baja comprensión lectora en algunos estudiantes. 


\section{Cuestionario a estudiantes}

Fue la técnica de recolección de información usada para indagar acerca de las estrategias que aplican los estudiantes para comprender un texto escrito.

\section{Procedimiento}

En la primera etapa se aplicaron en ambos grupos el instrumento CLP y el cuestionario a estudiantes; además, se realizó la entrevista a otros docentes de la institución.

En la segunda etapa se aplicó en el grupo de intervención una estrategia prediseñada de intervención pedagógica. Dicha propuesta se realizó con base en los tres momentos de la metacognición: planificación, monitoreo y evaluación del conocimiento (Baker \& Brown, 1984). Se desarrolló mediante intervenciones en el aula de clases durante el trabajo pedagógico regular, por medio de la aplicación de las guías interdisciplinares Me Pregunto y Comprendo (Meprecom), que usaban como eje transversal la pregunta guiada por el docente, antes, durante y después de la lectura de textos narrativos en el proceso de enseñanza de la Primera Lengua, de Ciencias Naturales, de Ciencias Sociales y de Matemáticas, para promover en los estudiantes el desarrollo de la estrategia metacomprensiva de la autopregunta. Esta se complementó con el uso de conocimientos previos, la predicción, el establecimiento de propósitos u objetivos y la verificación.

Finalmente, en la tercera etapa se evaluó el efecto de la intervención (cambios en el nivel de comprensión y en estrategias de metacomprensión en los estudiantes) con la aplicación del instrumento CLP luego de terminada la intervención en aula y de la aplicación del cuestionario a estudiantes a ambos grupos.

\section{Análisis de la información obtenida}

Con el fin de obtener información sobre las concepciones de los docentes de ciclo dos que no participaron como investigadores en este proyecto, sobre los procesos de comprensión lectora en los estudiantes a su cargo, se realizó un análisis de contenido de sus respuestas a la entrevista a otros docentes de la institución.
Uso de estrategias metacomprensivas para el fortalecimiento de la comprensión lectora

Bibian Alexandra Rodríguez R., María Eugenia Calderón S., Martha Helena Leal R. Nicolás Arias-Velandia

Para evaluar el grado de incorporación de estrategias de metacomprensión en los estudiantes, se compararon las respuestas de los estudiantes en el cuestionario a estudiantes de la evaluación inicial con las de la evaluación final. Adicionalmente, se consignaron observaciones espontáneas de los investigadores sobre los cambios que observaron en el desarrollo del trabajo con el grupo de intervención.

Para evaluar el efecto de la estrategia propuesta en este estudio sobre el nivel de comprensión lectora de los estudiantes, se analizaron los resultados de esta prueba en la evaluación inicial y en la evaluación final para determinar el cambio o la permanencia en el desempeño en la CLP. Esto se realizó mediante el análisis de la tendencia de medias y desviaciones típicas de los resultados de los estudiantes en ambos momentos (primera y tercera etapas), la comparación de muestras pareadas con la prueba $t$ de Student (paramétrica) y $W$ de Wilcoxon (no paramétrica) de los puntajes, dentro de cada grupo, entre la evaluación inicial y final. También se realizó a través de la comparación del número de estudiantes con puntuaciones por debajo de lo esperado en ambos grupos en la evaluación inicial y en la evaluación final.

Para garantizar la idoneidad del uso de la CLP, se calcularon los siguientes índices de validez y confiabilidad ${ }^{7}$ :

1. Análisis de la equivalencia entre niveles de evaluación de la prueba y niveles de formación en el sistema educativo colombiano manifestados en los Estándares básicos de competencias del Lenguaje del Ministerio de Educación Nacional de Colombia (2006). Se concluyó que existe equivalencia entre ambos criterios de evaluación del avance y desempeño de los estudiantes.

2. Verificación de tendencias de resultados comparando la muestra original de

7 Esta es una práctica habitual para examinar el uso idóneo de instrumentos construidos en otros contextos o con poblaciones diferentes a aquellas con las cuales se usan en cada investigación particular. Este procedimiento se realizó con los resultados obtenidos en la prueba CLP por todos los estudiantes (tanto del grupo de intervención como del grupo de comparación). 
Universidad Pedagógica Nacional

Facultad de Humanidades

validación y los resultados de este estudio. $\mathrm{Al}$ respecto, se encontró la misma tendencia de dificultad creciente en la muestra de validación de la prueba original realizada por Alliende, Condemarín \& Milicić (2004) con estudiantes del área metropolitana de Santiago de Chile y en los puntajes obtenidos por los estudiantes de esta investigación en la aplicación de la prueba en la evaluación inicial (véase el cuadro 3). La dificultad de la prueba aumenta (según se refleja en la disminución de la media de los puntajes a medida que se avanza en las pruebas de la 1 a la 4 en el subtest III) y la tendencia es relativamente constante en la desviación típica de las dos poblaciones.

Cuadro 2. Tendencias de dificultad en las puntuaciones obtenidas en la muestra original de validación de la prueba en la evaluación previa en el presente trabajo

\begin{tabular}{|l|l|l|}
\hline \multicolumn{1}{|c|}{ Subprueba } & $\begin{array}{c}\text { Media y desvia- } \\
\text { ción en la mues- } \\
\text { tra de validación } \\
\boldsymbol{\mu ( \delta )}\end{array}$ & $\begin{array}{c}\text { Media y desvia- } \\
\text { ción en la eva- } \\
\text { luación previa } \\
\boldsymbol{\mu ( \delta )}\end{array}$ \\
\hline Subtest III (1) & $0,90(0,31)$ & $0,94(0,38)$ \\
\hline Subtest III (2) & $0,68(0,44)$ & $0,7114(0,45)$ \\
\hline Subtest III (3) & $0,65(0,43)$ & $0,6775(0,49)$ \\
\hline Subtest III (4) & $0,56(0,49)$ & $0,5366(0,49)$ \\
\hline
\end{tabular}

3. Utilización del grado de equivalencia o distancia entre puntuaciones $Z^{8}$ en la prueba de estandarización (Santiago, Chile) y la evaluación inicial en esta investigación como criterio de confiabilidad de la prueba.

Para establecer si había diferencias significativas entre las puntuaciones $\mathrm{Z}$ de los estudiantes de tercer

8 La puntuación Z es un cálculo para reducir cualquier distribución de puntajes a una escala que tiene como media o promedio $0 \mathrm{y}$ desviación estándar entre 1 y-1. Resulta de restar a cada puntuación el valor de la media o del promedio, y de dividir dicho resultado entre el valor de la desviación estándar. De este modo, los resultados obtenidos para cada puntuación muestran que tan lejos está cada puntuación de la media (del 0 en Z) y de las desviaciones estándar (de las puntuaciones Z de 1 y de -1) (Hernández-Sampieri et.al., 2010). grado y las del mismo grado en la muestra de validación, se calculó el estadístico prueba $t$ de Student de comparación entre muestras independientes, asumiendo varianzas distintas, con dos colas. Este nos arrojó como resultado una probabilidad de tener diferencias entre las puntuaciones $Z$ en ambas distribuciones de 0,4003 . Si bien no es una probabilidad pequeña, no muestra diferencias significativas entre las puntuaciones $\mathrm{Z}$ de la muestra original de validación (Alliende et al., 2004) y las de los estudiantes en evaluación previa en el presente trabajo. Por lo tanto, se asume que ambas distribuciones son equivalentes y que la prueba tiene suficientes índices de confiabilidad en los estudiantes de tercer grado del Colegio Alfredo Iriarte IED.

Para establecer posibles diferencias significativas entre las puntuaciones $\mathrm{Z}$ entre los estudiantes de cuarto grado de este estudio y los de la muestra original de validación, se utilizó nuevamente el estadístico prueba $t$ de Student de comparación entre muestras independientes, asumiendo varianzas distintas, con dos colas. Este cálculo tuvo como resultado una probabilidad de tener diferencias entre las puntuaciones $\mathrm{Z}$ en ambas distribuciones de 0,2398 . Esto muestra una probabilidad pequeña $y$ ausencia de diferencias significativas entre las puntuaciones $\mathrm{Z}$ de la muestra original de validación y las de los estudiantes en evaluación previa en el presente trabajo en cuarto grado.

\section{Resultados}

Los resultados que se presentan a continuación guardan el siguiente orden:

- Presentación de resultados obtenidos del instrumento CLP sobre el nivel de comprensión lectora en los estudiantes.

- Análisis de la evolución en las estrategias metacomprensivas de los estudiantes que participaron en el grupo de intervención.

- Un compendio de las observaciones adicionales sobre la aplicación de la estrategia Meprecom, por parte de los investigadores. 
- Presentación de ideas manifiestas de los docentes sobre comprensión lectora y aspectos relacionados, al preguntarles por esto en un solo momento antes de la intervención probada en este estudio.

A continuación se desarrolla cada uno de estos grupos de resultados obtenidos.
Uso de estrategias metacomprensivas para el fortalecimiento de la comprensión lectora

Bibian Alexandra Rodríguez R., María Eugenia Calderón S., Martha Helena Leal R. Nicolás Arias-Velandia

Nivel de comprensión lectora en los estudiantes

\section{Resultados generales de comprensión lectora}

El instrumento CLP se aplicó para evaluar la comprensión lectora de los estudiantes antes y después del trabajo de aula en estrategias metacomprensivas. En el cuadro 4 se pueden observar las tendencias de los puntajes obtenidos por los estudiantes de ambos grupos.

Cuadro 3. Tendencias en puntuaciones en la prueba CLP

\begin{tabular}{|c|c|c|c|c|c|c|c|}
\hline \multirow{2}{*}{ GRUPO } & \multirow[t]{2}{*}{ Subsubtest } & \multicolumn{3}{|c|}{$\begin{array}{c}\text { Pre: } \\
\text { PrIMERA APLICACIÓN }\end{array}$} & \multicolumn{3}{|c|}{$\begin{array}{c}\text { Post: } \\
\text { SEgUNDA APLICACIÓN }\end{array}$} \\
\hline & & Suma & Promedio & Desviación & Suma & Promedio & Desviación \\
\hline \multirow{4}{*}{$\begin{array}{l}\text { Intervención } \\
\text { GRADO } 3 \text { SEDE C SUBTEST } 3 \text { FORMA A }\end{array}$} & Subsubtest 1 & 19,43 & 0,75 & 0,42 & 24,57 & 0,94 & 0,16 \\
\hline & Subsubtest 2 & 16,83 & 0,67 & 0,43 & 21,17 & 0,81 & 0,37 \\
\hline & Subsubtest 3 & 15 & 0,6 & 0,47 & 22,2 & 0,85 & 0,28 \\
\hline & Subsubtest 4 & 12,33 & 0,49 & 0,5 & 15,33 & 0,59 & 0,49 \\
\hline \multirow{4}{*}{$\begin{array}{l}\text { Intervención } \\
\text { GRADO } 4 \text { SEDE C SUBTEST } 3 \text { FORMA B }\end{array}$} & Subsubtest 1 & 19 & 0,83 & 0,38 & 23 & 0,98 & 0,04 \\
\hline & Subsubtest 2 & 14 & 0,61 & 0,46 & 19 & 0,83 & 0,37 \\
\hline & Subsubtest 3 & 10,25 & 0,44 & 0,49 & 12,5 & 0,54 & 0,5 \\
\hline & Subsubtest 4 & 8,33 & 0,36 & 0,49 & 12 & 0,52 & 0,51 \\
\hline \multirow{4}{*}{$\begin{array}{l}\text { Comparación } \\
\text { GRADO } 3 \text { SEDE B SUBTEST } 3 \text { FORMA A }\end{array}$} & Subsubtest 1 & 12 & 0,86 & 0,35 & 11,71 & 0,84 & 0,37 \\
\hline & Subsubtest 2 & 7 & 0,5 & 0,41 & 7 & 0,5 & 0,48 \\
\hline & Subsubtest 3 & 7 & 0,5 & 0,41 & 7,4 & 0,53 & 0,51 \\
\hline & Subsubtest 4 & 6 & 0,43 & 0,47 & 6,67 & 0,48 & 0,51 \\
\hline \multirow{4}{*}{$\begin{array}{l}\text { Comparación } \\
\text { GRADO } 4 \text { SEDE B SUBTEST } 3 \text { FORMA B }\end{array}$} & Subsubtest 1 & 17 & 0,85 & 0,32 & 17,85 & 0,79 & 0,33 \\
\hline & Subsubtest 2 & 13,14 & 0,66 & 0,39 & 12,71 & 0,63 & 0,41 \\
\hline & Subsubtest 3 & 9,25 & 0,46 & 0,49 & 9,5 & 0,47 & 0,5 \\
\hline & Subsubtest 4 & 8,33 & 0,42 & 0,5 & 8,33 & 0,42 & 0,5 \\
\hline
\end{tabular}

La suma, el promedio y la desviación obtenida en los resultados arrojados por el grupo de intervención en la evaluación final, en contraste con los obtenidos en la evaluación inicial, dieron cuenta de una reducción de la distancia en el desempeño de los estudiantes y de un mayor desarrollo de las habilidades en comprensión lectora indagadas por la prueba en el subtest 3 en sus formas A y B en la evaluación final (véase el cuadro 3). Estas habilidades son:

- Interpretar el sentido de una oración o frase leída.

- Seguir instrucciones escritas para comprender el sentido de un conjunto de frases u oraciones identificando los sujetos de las acciones y las cualidades asignadas a los nombres. 
Universidad Pedagógica Nacional

Facultad de Humanidades

- Comprender el sentido general de un texto mediante la elaboración de inferencias básicas de hechos no explícitos en una sola frase, sino que corresponden al sentido general de lo narrado.

- Comprender una expresión o idea compleja con ayuda de una explicación.

En cuanto al grupo de comparación, Sede B, al aplicarle la prueba CLP en la evaluación final para analizar nuevamente el estado comprensivo lector de sus estudiantes, se observó que los promedios y las desviaciones típicas que se obtienen son similares a los de la evaluación inicial (véase el cuadro 3). Este desempeño muestra una tendencia más baja que la del grupo de intervención.

Los anteriores resultados se confirmaron con las pruebas estadísticas. La comparación de muestras pareadas de las puntuaciones en la evaluación inicial y en la evaluación final dentro de cada grupo (control e intervención) con la prueba no paramétrica W de Wilcoxon no mostró diferencias significativas en el grupo de comparación, $Z=-1,088$, Sig. Asint. $=0,277$, y sí mostró diferencias significativas en el grupo de intervención, $Z=-5,936$, Sig. Asint. $=0,000$. En el mismo sentido, la comparación de muestras independientes entre grupos control e intervención se realizó con la suma de puntajes en la evaluación inicial utilizando la prueba estadística $t$ de Student para la comparación de muestras independientes, no mostró diferencias significativas en las puntuaciones de los grupos en la evaluación previa, F Levene $=5,256$, Leven Sig $=0,024 ; \mathrm{t}=0,190$, $\mathrm{gl}=80,998$, Sig $(2$ colas $)=0,849$. Por otra parte, la prueba no paramétrica U de Mann-Whitney para la comparación de muestras independientes mostró diferencias significativas entre los puntajes del grupo de comparación y el grupo de intervención en la evaluación final, $U=298,000, Z=-4,984$, Sig. Asint. $=0,000$.

\section{Resultados de desempeño menor al esperado. Los cuadros 4 y 5 muestran el número y porcentaje de estudiantes con puntuaciones por debajo de lo esperado para los criterios del instrumento CLP}

Cuadro 4. Número de estudiantes de tercer grado con resultados por debajo de lo esperado en el instrumento CLP

\begin{tabular}{|l|c|c|}
\hline \multicolumn{1}{|c|}{ Etapa } & $\begin{array}{c}\text { Grupo de } \\
\text { intervención } \\
\text { Tercer grado } \\
\text { (26 Estudiantes) }\end{array}$ & $\begin{array}{c}\text { Grupo de } \\
\text { comparación } \\
\text { Tercer grado } \\
\text { (14 Estudiantes) }\end{array}$ \\
\hline $\begin{array}{l}\text { CLP evaluación } \\
\text { inicial }\end{array}$ & $\begin{array}{c}42,30 \% \\
\text { (11 estudiantes) }\end{array}$ & $\begin{array}{c}50 \% \\
\text { (7 estudiantes) }\end{array}$ \\
\hline $\begin{array}{l}\text { CLP evaluación } \\
\text { final }\end{array}$ & $\begin{array}{c}8 \% \\
\text { (2 estudiantes) }\end{array}$ & $\begin{array}{c}50 \% \\
\text { (7 estudiantes) }\end{array}$ \\
\hline
\end{tabular}

En la segunda aplicación de la prueba CLP, en el grupo de intervención del tercer grado disminuyó el porcentaje de estudiantes que se encontraban por debajo de lo esperado en su desarrollo, y en el grupo de comparación del mismo grado permaneció igual a la primera aplicación (véase el cuadro 4). También se puede observar que en la segunda aplicación de la prueba CLP en cuarto grado (véase el cuadro 5), el porcentaje de estudiantes que se encontraba por debajo de lo esperado en el grupo de intervención disminuyó y en el grupo de comparación aumentó.

Cuadro 5. Número de estudiantes de cuarto grado con resultados por debajo de lo esperado en el instrumento CLP

\begin{tabular}{|l|c|c|}
\hline \multicolumn{1}{|c|}{ Etapa } & $\begin{array}{c}\text { Grupo de } \\
\text { intervención } \\
\text { Grado 4 } \\
\text { (23 Estudiantes) }\end{array}$ & $\begin{array}{c}\text { Grupo de } \\
\text { comparación } \\
\text { Grado 4 } \\
\text { (20 Estudiantes) }\end{array}$ \\
\hline $\begin{array}{l}\text { CLP evaluación } \\
\text { inicial }\end{array}$ & $\begin{array}{c}39,13 \% \\
\text { (9 estudiantes) }\end{array}$ & $\begin{array}{c}20 \% \\
\text { (4 estudiantes) }\end{array}$ \\
\hline $\begin{array}{l}\text { CLP evaluación } \\
\text { final }\end{array}$ & $\begin{array}{c}13 \% \\
\text { (3 estudiantes) }\end{array}$ & $\begin{array}{c}30 \% \\
\text { (6 estudiantes) }\end{array}$ \\
\hline
\end{tabular}

\section{Evolución en las estrategias metacomprensivas de los estudiantes}

El cuadro 6 muestra los resultados de la frecuencia de respuesta y del porcentaje de estrategias metacomprensivas de los estudiantes según el cuestionario a Estudiantes, antes y después del trabajo en aula con Meprecom. Estos resultados muestran que entre la evaluación inicial y la evaluación final aumenta el porcentaje de estudiantes que manifiesta hacer preguntas antes, durante y después de la lectura de textos escritos. También aumenta el porcentaje de estudiantes que manifiestan usar estrategias como la predicción, el subrayado, releer y tener en cuenta los conocimientos previos. 


\begin{tabular}{|c|c|c|c|c|c|c|c|c|c|c|c|c|c|c|c|c|c|c|c|c|c|c|c|c|c|c|c|}
\hline \multirow{2}{*}{ 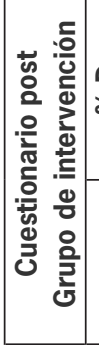 } & 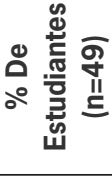 & $\underset{+\infty}{\infty}$ & 咅 & $\begin{array}{l}\hat{N} \\
\tilde{j} \\
\tilde{\theta}\end{array}$ & $\begin{array}{l}0 \\
\stackrel{0}{\infty} \\
\end{array}$ & \begin{tabular}{|l}
$\infty$ \\
$\infty$ \\
- \\
-1
\end{tabular} & $\begin{array}{l}\Omega \\
\infty \\
0 \\
0 \\
\end{array}$ & 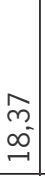 & 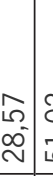 & & $\begin{array}{l}\vec{n} \\
\text { Sू } \\
\end{array}$ & $\mid \begin{array}{c}\stackrel{\sim}{m} \\
\hat{\omega} \\
\sigma\end{array}$ & 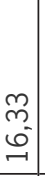 & 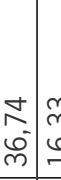 & & $\begin{array}{l}\text { s. } \\
\text { s. } \\
\text { m. }\end{array}$ & 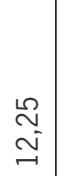 & $\begin{array}{l}\text { ŏ } \\
\text { స̆ }\end{array}$ & $\underset{\sim}{\stackrel{d}{*}}$ & $\begin{array}{l}\vec{f} \\
\vdots \\
\vdots \\
\vdots\end{array}$ & 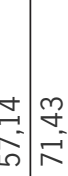 & $\begin{array}{l}\infty \\
\infty \\
\bar{\sigma}\end{array}$ & 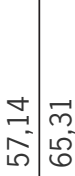 & $\begin{array}{l}\infty \\
\infty \\
\infty \\
\infty\end{array}$ & 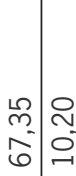 & 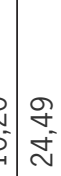 & $\begin{array}{l}\overrightarrow{6} \\
\dot{m} \\
\dot{m}\end{array}$ \\
\hline & 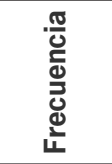 & $\sim$ & 으 & $\vec{m}$ & $\theta$ & $\sim$ & i & $a$ & $\exists$ & $\stackrel{\sim}{\sim}$ & $\hat{m}$ & m & $\infty$ & $\stackrel{\infty}{\rightarrow} \mid \alpha$ & & $\nexists$ & 0 & $\stackrel{\infty}{+}$ & $\rightarrow$ & 응ㅇㅇ & $\left.\begin{array}{c}\infty \\
\sim\end{array}\right)$ & L & $\stackrel{\infty}{\sim} \underset{m}{m}$ & 寸 & مـ & $\approx$ & $\stackrel{\Omega}{\sim}$ \\
\hline 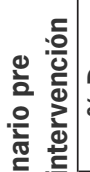 & 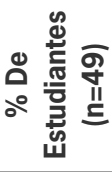 & 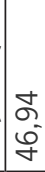 & $\begin{array}{l}\stackrel{2}{N} \\
\dot{ت}\end{array}$ & 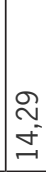 & 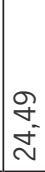 & 0 & $\begin{array}{l}\stackrel{g}{+} \\
\dot{ \pm} \\
\sim\end{array}$ & 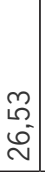 & $\begin{array}{ll}\overrightarrow{0} & \\
0 \\
0\end{array}$ & 足 & $\begin{array}{l}\hat{n} \\
\infty^{\infty} \\
\sim\end{array}$ & 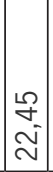 & 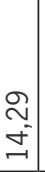 & 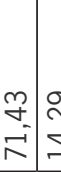 & 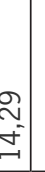 & 0 & 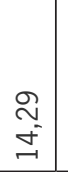 & $\begin{array}{l}\infty \\
\stackrel{\infty}{ } \\
\infty \\
\infty\end{array}$ & $\begin{array}{l}\tilde{\delta} \\
\bar{n}\end{array}$ & 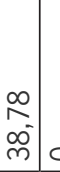 & \begin{tabular}{l|l}
$\vec{b}$ \\
0 \\
0
\end{tabular} & 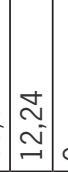 & 0 & 0 & 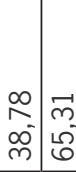 & $\begin{array}{l}8 \\
8 \\
\end{array}$ & 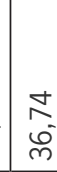 \\
\hline 胥 & $\begin{array}{l}\overrightarrow{\widetilde{E}} \\
\text { ष्ँ }\end{array}$ & $\stackrel{m}{\sim}$ & N & & $\cong$ & 0 & $\cong$ & $\stackrel{m}{\rightarrow}$ & צ & \begin{tabular}{ll|l}
0 & \\
-1 &
\end{tabular} & $a$ & $\exists$ & $\wedge$ & $\stackrel{m}{m}$ & & 0 & 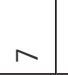 & $\stackrel{\Delta}{\sim}$ & $\stackrel{\sim}{\sim}$ & $\stackrel{\Omega}{\triangle}$ & مـ & 0 & 00 & 0 & 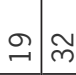 & $\approx$ & $\stackrel{\infty}{\rightarrow}$ \\
\hline
\end{tabular}

\section{竞 寸}

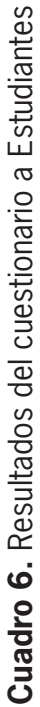

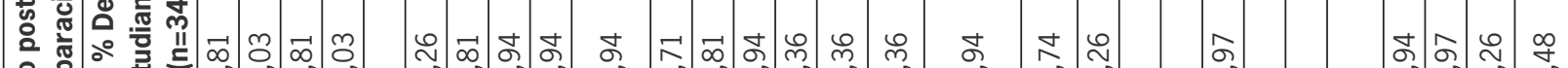

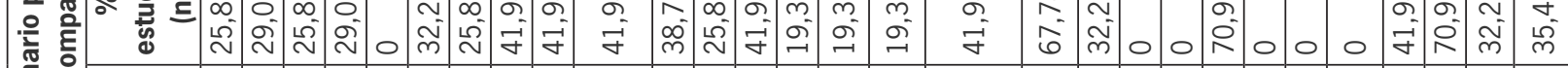

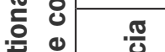

을

要

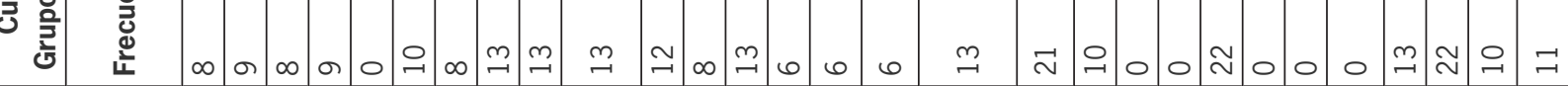
:흥

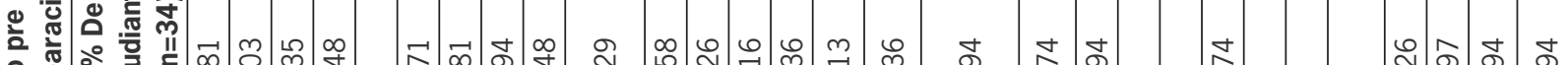
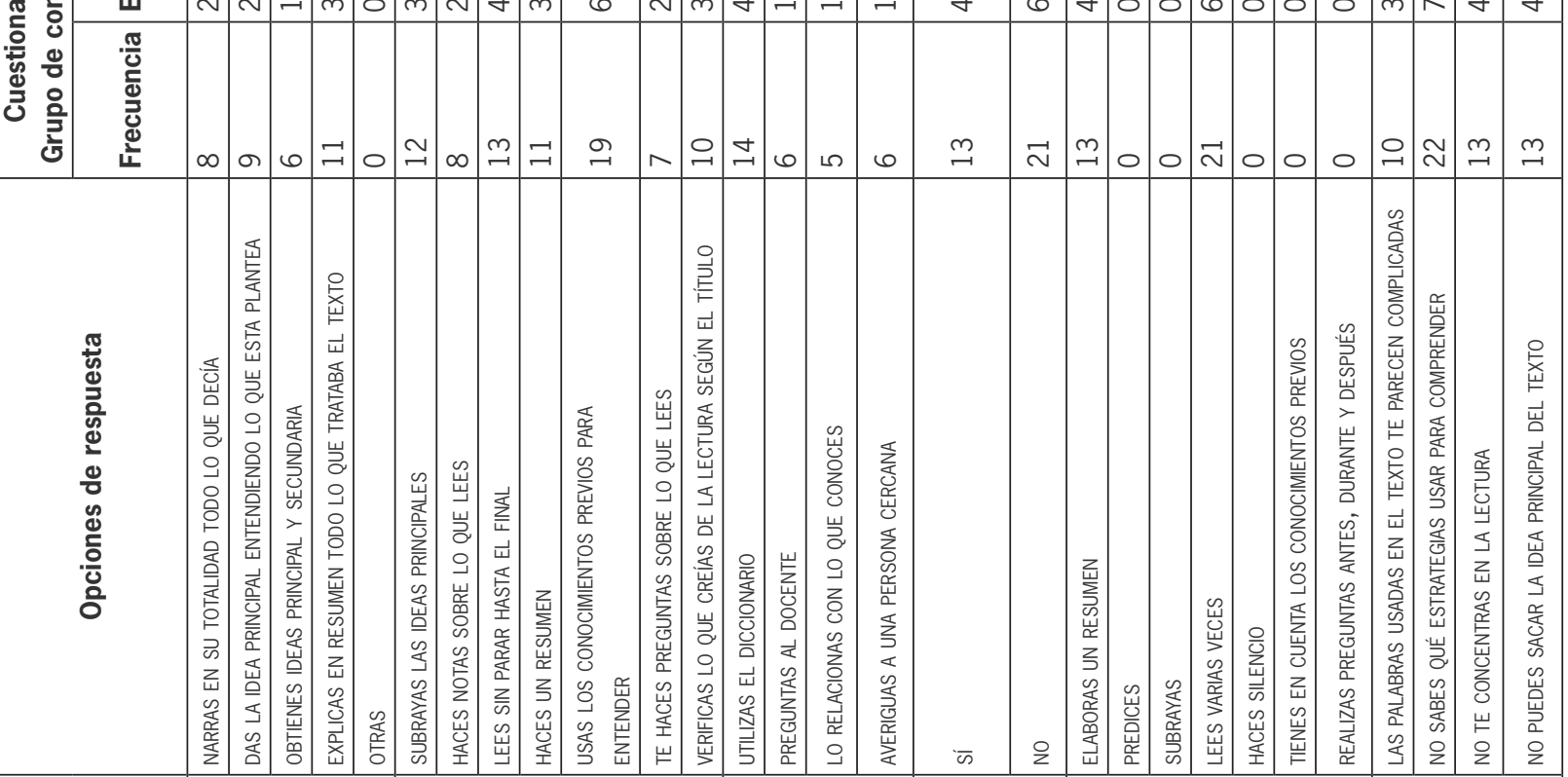

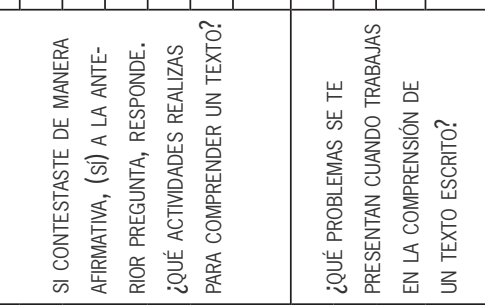


Del mismo modo, antes de la intervención las respuestas más frecuentes de los estudiantes a cuándo comprenden un texto son las de recuperar todo lo que el texto dice o resumirlo en su totalidad. Al finalizarla, los estudiantes objeto de intervención plantean respuestas sobre identificar idea principal y encontrar ideas secundarias (véase el cuadro 6).

Entre las estrategias manifestadas para comprender lo que leen, al inicio de la intervención los estudiantes mencionan casi indistintamente cualquier estrategia, y al final de la misma, sus respuestas más frecuentes se concentran en subrayar ideas principales, hacer resúmenes y realizar preguntas antes, durante y después de la lectura. La tendencia contraria se presenta cuando se pregunta por lo que hacen al encontrar palabras desconocidas: antes de la intervención en aula solamente plantean buscarlas en el diccionario, $y$ al final de la misma plantean casi con igual frecuencia buscar en el diccionario, indagar con el docente $u$ otras personas cercanas, $y$ relacionar con lo que ya conocen (véase el cuadro 6).

En el cuadro 6 también se muestra que al inicio un poco menos de la mitad de los estudiantes manifestaba que las estrategias les ayudaban a entender el texto y casi la totalidad al final manifiestan que dichas estrategias les ayudan a comprender los textos. Asimismo, al manifestar cuáles aspectos les cusan dificultades en esta comprensión, expresan antes de la intervención en aula que son casi indistintamente todas las opciones, con un cierto pre- dominio de no tener estrategias para comprender, mientras que al finalizar dicho proceso la mayor dificultad manifiesta de los estudiantes se concentra en la aparición de palabras o vocabulario "complicado" (véase el cuadro 6).

\section{Observaciones adicionales sobre la aplicación de la estrategia Meprecom}

Además de los resultados anteriores, los investigadores observaron que los estudiantes que aplicaron la estrategia Meprecom manifestaron mayor gusto y motivación al participar en actividades de comprensión lectora, incorporaron como rutina las actividades de planeación, verificación y evaluación al buscar comprender textos y el uso de autopreguntas como herramienta principal en ellas. También advierten que varias de las estrategias suelen aparecer más cuando leen textos narrativos que con otros tipos de texto, con lo cual se subraya la necesidad de diversificar los tipos de texto utilizados en la actividad para evitar limitaciones artificiales en la aplicación de las estrategias.

\section{Ideas manifiestas de los docentes sobre la comprensión lectora y aspectos relacionados}

En la tabla 3 se muestran las respuestas dadas por ocho de los doce docentes (diferentes a los investigadores) que trabajan con el ciclo dos en el Colegio Distrital Alfredo Iriarte.

Tabla 3. Respuestas de los docentes de ciclo dos del Colegio Distrital Alfredo Iriarte sobre comprensión lectora

\begin{tabular}{|c|c|c|}
\hline $\begin{array}{l}\text { ¿QUÉ ENTIENDES POR } \\
\text { COMPRENSIÓN LECTORA? }\end{array}$ & $\begin{array}{l}\text { ¿QUÉ ESTRATEGIAS UTILIZAS EN } \\
\text { EL AULA PARA DESARROLLAR } \\
\text { LA COMPRENSIÓN EN } \\
\text { LOS ESTUDIANTES? }\end{array}$ & $\begin{array}{l}\text { ¿CUÁLES CREES QUE SON LAS PRINCIPALES } \\
\text { CAUSAS DE QUE LOS NIÑOS PRESENTEN } \\
\text { UNA BAJA COMPRENSIÓN LECTORA? }\end{array}$ \\
\hline $\begin{array}{l}\text { Capacidad de analizar, inferir y } \\
\text { argumentar. } \\
\text { Interactuar con el texto. } \\
\text { Habilidad para analizar e interpretar. } \\
\text { Capacidad de extraer información. } \\
\text { Capacidad de sintetizar. }\end{array}$ & $\begin{array}{l}\text { Realización de lectura en voz alta. } \\
\text { Contextualizar, después de leer. } \\
\text { Lecturas de interés del estudiante. } \\
\text { Interpretación grafica de lo leído. } \\
\text { Lecturas dirigidas y grupales. } \\
\text { Creación de cuentos grupales a partir de } \\
\text { lecturas. } \\
\text { Lectura entre pares. } \\
\text { Lectura de diversos géneros literarios. }\end{array}$ & $\begin{array}{l}\text { Se les dificulta extraer ideas. } \\
\text { Poco contacto con los textos. } \\
\text { Metodologías tradicionales y obsoletas. } \\
\text { Falta de tiempo para leer en el aula. } \\
\text { Poca motivación del docente y el estudiante por la } \\
\text { lectura. } \\
\text { Actividades de lectura sin sentido. } \\
\text { No existen hábitos de lectura: ni en casa ni en la } \\
\text { escuela. } \\
\text { No comprender aspectos formales y conceptuales } \\
\text { del texto leído. } \\
\text { Invasión de las tecnologías. } \\
\text { No se establecen relaciones de interdisciplinariedad. }\end{array}$ \\
\hline
\end{tabular}


Las respuestas de los docentes en la tabla 3 muestran que quienes respondieron atribuyen al concepto de comprensión lectora habilidades como interpretar o dar sentido. También se reflejan otras respuestas que relacionan la comprensión lectora con analizar, inferir y argumentar. Entre las estrategias que manifiestan para trabajar la comprensión lectora se nombran actividades de análisis como contextualizar o interpretar imágenes, actividades de motivación como lecturas en voz alta o lecturas de interés del estudiante y actividades de dinámica grupal como lectura dirigida, grupal, creación de cuentos grupalmente o lectura entre pares. Al evocar situaciones que inciden en bajos niveles de comprensión lectora de estudiantes de su ciclo, atribuyen esto a dificultades personales del estudiante (para extraer ideas, aspectos formales del texto o para establecer relaciones), en otros casos a factores del contexto (poco contacto con los textos, lectura sin sentido, falta de lectura en casa y escuela, o invasión tecnológica) o incluso a actividades de los mismos docentes (fallas al intentar motivar a los estudiantes, uso de metodologías tradicionales y obsoletas de enseñanza). Por otra parte, estas respuestas de docentes de ciclo dos de la institución que no son investigadores en este proceso no incluyen estrategias de conocimiento de los estudiantes sobre su propio proceso comprensivo para poder autorregularlo.

\section{Discusiones}

Los resultados de indagación con otros docentes de segundo ciclo del Colegio Alfredo Iriarte sobre sus ideas del trabajo de comprensión lectora no evidencian que tengan en cuenta el uso de estrategias metacomprensivas en sus prácticas pedagógicas. Esto hace necesario repensar el papel que los docentes están concediendo al estudiante dentro del proceso de aprendizaje: al permitirle desarrollar un proceso metacognitivo en la lectura, el educando tendrá un rol más activo y aprenderá a aprender (Burón, 1999; Pinzas, 2003) conociendo el proceso y las estrategias necesarias para desarrollarlo.
Uso de estrategias metacomprensivas para el fortalecimiento de la comprensión lectora

Bibian Alexandra Rodríguez R., María Eugenia Calderón S., Martha Helena Leal R Nicolás Arias-Velandia

Varias investigaciones previas (Adrianzén, 2012; Baker \& Brown, 1984; Condori, 2003; FlórezRomero et al., 2005; Heit, 2012; Irrazabal et al., 2006; Nieto, 2005; Quaaset et al., 2005; Santibáñez \& Pisconte, 2003; Wong \& Matalinares, 2011) mostraron que el trabajo con estrategias metacognitivas o metacomprensivas en comprensión lectora mejora el desempeño de los estudiantes en dicha comprensión. El presente estudio muestra que, además de la utilización de estas estrategias y de su combinación, su uso teniendo como eje la autopregunta tiene efectos en el mejoramiento de desempeño en comprensión lectora en los estudiantes (como se evidencia en los resultados con la prueba CLP) y en incorporar nuevos elementos de metacomprensión en la lectura (como evidencian los cuestionarios hechos a los estudiantes que participaron de estas estrategias). Por ese motivo, el análisis de estos aspectos ya había sido sugerido previamente por Mateus, Santiago, Castillo y Rodríguez (2012).

De otra parte, las estrategias metacognitivas y metacomprensivas se pueden abordar desde el concepto de zona de desarrollo próximo (ZDP) de Vigotsky (1989), porque al igual que esta, actúan como un puente que se configura en un andamiaje (Bruner, 1999) que permite al estudiante desarrollar apoyos internos que lo llevan a transitar de los procesos ya consolidados a los procesos en elaboración (Vigotsky, 1989). Al respecto, Mateos (1999) plantea que la metacognición, entendida como autocontrol o autorregulación de la actividad, es un aspecto fundamental tanto en aprendices expertos como en novatos: aunque los aprendices expertos tienden a hacer la actividad de forma automática, en problemas poco conocidos o en ámbitos diferentes a los ya conocidos deben emplear la regulación consciente y sistemática de la actividad. Por su parte, los novatos para obtener buenos resultados en la actividad cognoscente deben planear, controlar y supervisar las estrategias utilizadas, identificando las deficiencias en la comprensión y en las operaciones realizadas.

En relación con la discusión anterior, las estrategias metacognitivas y metacomprensivas plantean demandas altamente exigentes a los estudiantes, lo 
Universidad Pedagógica Nacional

Facultad de Humanidades

cual puede despertar dudas sobre la capacidad de los niños de tercer y cuarto grados de primaria para llevarlas a cabo. Sin embargo, R. Puche Navarro, M. Orozco, B. C. Orozco y M. Correa (2009) exponen que los niños ya desde el periodo de los tres a cinco años evidencian una primera comprensión compleja de estados psicológicos de otras personas, usando activamente herramientas de conocimiento como la clasificación, la planeación, la predicción o anticipación, la inferencia y la formulación de hipótesis. Según Flavell (1976) y Brown (1980) estas herramientas serían primeras evidencias del desarrollo de un proceso metacognitivo. Además, Tolchinsky y Simó (2003) muestran que el uso de dichas herramientas ya es provechoso en estudiantes de todos los grados en la escuela primaria o elemental.

En cuanto a la potenciación de las habilidades de comprensión lectora, Adrianzén (2012), FlorézRomero et al. (2005), Irrazabal et al. (2006) y Silvestri (2006) dan cuenta del efecto potenciador que la aplicación de estrategias metacomprensivas genera en el desarrollo comprensivo lector de los estudiantes. La presente investigación también apoya esta conclusión. El efecto potenciador que el desarrollo de las habilidades metacomprensivas genera en el proceso comprensivo lector permite proponerlas como un puente entre los diferentes niveles que componen la comprensión lectora de tipo literal, de retención, de organización, inferencial, de interpretación, de valoración y de creación, propuestos por autores como Sánchez (2009). Se plantea entonces la necesidad de implementar estas estrategias a mayor escala en el Colegio Alfredo Iriarte, con el fin de incorporar en ellas en el futuro a otros docentes que han participado respondiendo encuestas y cuestionarios de esta investigación pero que han continuado con sus prácticas habituales de enseñanza de la comprensión lectora con estudiantes de segundo ciclo.

En el momento de trabajar en la comprensión de textos es común encontrar que resulta más fácil interpretar y comprender un texto narrativo (cuento, mito, leyenda, fábula), ya que estos sitúan al estudiante en un tiempo, con unos personajes, unos escenarios y unos acontecimientos organizados (Bruner, 1999). Sin embargo, cuando enfrenta la lectura y comprensión de otro tipo de textos, el estudiante no necesariamente comprende la lógica de tales textos y por eso no supone que los mismos recursos usados con los textos narrativos sirven para comprender textos no narrativos (Pérez, 2003; Tolchinsky \& Simó, 2003).

Por ese motivo debe continuar el enriquecimiento del programa de intervención en metacomprensión introduciendo en este el trabajo específico de reconocimiento de diferentes tipos de texto. Esto es posible, y además abre otra línea de trabajo posterior con textos no narrativos, ya que las estrategias metacomprensivas tratadas en este estudio pueden extenderse a otro tipo de textos, pero deben ser ejercitadas con esos tipos de texto para asegurar la transferencia en el aprendizaje de la comprensión lectora y de estas estrategias a los diferentes tipos de texto que puede enfrentar en su historia educativa.

\section{Referencias bibliográficas}

Adrianzén, G. V. (2012). Aplicación de un programa de habilidades metacognitivas para mejorar la comprensión lectora en niños de 4to. grado de primaria del Colegio Parroquial Santísima Cruz de Chulucanas. Piura: Repositorio Institucional Pirhua. Universidad de Piura.

Alliende, F.; Contemarin, M. \& Milicić, N. (2004). Prueba CLP Formas Paralelas. Manual para la aplicación de la prueba de comprensión lectora de complejidad lingüística progresiva, 8 niveles de lectura. 7. ${ }^{\text {ed. Santiago, }}$ Chile: Pontificia Universidad Católica.

Baker, L. \& Brown, A. L. (1984). Metacognitive skills in reading. En P. D. Pearson (ed.), Handbook of reading research (pp. 353-394). Nueva York: Longman.

Blythe, T. \& Perkins, D. (1999). Comprender la comprensión. En T. Blythe (comp.). La enseñanza para la comprensión. Guía para el docente. Buenos Aires: Paidós.

Brown, A. (1980). Metacognitive development and reading. En R. J. Spiro, B. C. Bruce \& F. Brewer (eds.). Theoretical issues in reading comprehension (pp. 453-481). Hillsdale, NJ: Erlbaum.

Bruner, J. S. (1999). The culture of education. Cambridge, Mass: Harvard University Press. 
Burón, J. (1999). Enseñar a aprender: introducción a la metacognición. Bilbao: Mensajero.

Cassany, D. (2006). Tras las líneas. Sobre la lectura contemporánea. Barcelona: Anagrama.

Ministerio de Educación Nacional (2006). Estándares básicos de competencias del Lenguaje, Matemáticas, Ciencia y Ciudadanas. Bogotá: autor.

Condori, L. S. (2003). Aplicación de estrategias metacognitivas para mejorar la comprensión lectora en los alumnos de de cuarto grado " $B$ " de la institución educativa pública N. ${ }^{\circ} 70537$ del distrito de Cabanillas de la provincia de San Román del departamento de Puno. Lima, Perú.

Cuetos, F.; Rodríguez, B. \& Ruano, E. (2004) Prolec. Evaluación de los procesos lectores. 5. ${ }^{\mathrm{a}}$ ed. Madrid: TEA Ediciones.

Dubois, M. E. (2000). El proceso de lectura: de la teoría a la práctica. Buenos Aires: Aique.

Flavell, J. H. (1976). Metacognitive aspects of problem solving. En L. B. Resnick (ed.), The nature of intelligence (pp. 231-235). Hillsdale, NJ: Lawrence Erlbaum.

Flavell, J. H.; Baker, L. \& Brown, A. L. (1984). Metacognitive skills and reading. En P. D. Pearson, R. Barr, M. L. Kamil \& P. Mosenthal (eds.). Handbook of reading research (pp. 353-394). Mahwah, NJ: Lawrence Erlbaum.

Flórez-Romero, R.; Arias-Velandia, N. \&Torrado, M. C. (2006). Leer en familia: horizonte conceptual. En Fundalectura (ed.). Leer en Familia en Colombia: reporte de investigación y experiencias (pp. 19 - 45). Bogotá: Fundalectura.

Flórez-Romero, R; Torrado, M. C.; Arévalo-Rodríguez, I.; Mesa, C.; Mondragón, S. \& Pérez, C. (2005). Habilidades metalingüísticas, operaciones metacognitivas y su relación con los niveles de competencia en lectura y escritura: un estudio exploratorio. Forma y Función, 18, 15-44.

Flórez-Romero, R.; Restrepo, M. A. \& Schwanenflugel, P. (2007). Alfabetismo emergente, investigación, teoría y práctica. Bogotá: Departamento de Comunicación Humana, Facultad de Medicina, Universidad Nacional de Colombia - Instituto para la Investigación Educativa y el Desarrollo Pedagógico (IDEP).

Gil-Chaves, L. \& Flórez-Romero, R. (2011). Desarrollo de habilidades de pensamiento inferencial y comprensión de lectura en niños de tres a seis años. Panorama, 5 (9), 103- 125.
Uso de estrategias metacomprensivas para el fortalecimiento de la comprensión lectora

Bibian Alexandra Rodríguez R., María Eugenia Calderón S., Martha Helena Leal R Nicolás Arias-Velandi

Graesser, A. C.; Singer, M. \& Trabasso, T. (1994). Constructing inferences during narrative text comprehension. Psychological Review, 101 (3), 371-395.

Heit, I. A. (2012). Estrategias metacognitivas de comprensión lectora y eficacia en la asignatura Lengua y Literatura. Revista de Psicología, 8 (15), 79-96.

Hernández-Sampieri, R., Fernández Collado, C. \& Baptista Lucio, P. (2010). Metodología de la investigación. México: McGraw-Hill.

Irrazabal, N.; Saux, G., Burin, D. \& León, J. A. (2006). El resumen: comprensión lectora en estudiantes universitarios. Memorias de las XiII Jornadas de Investigación, Facultad de Psicología, Universidad de Buenos Aires, 2.

Mateus, G. E.; Santiago, A. W.; Castillo, M. C. \& Rodríguez, L. G. (2012). Lectura y representación mental de textos expositivos en estudiantes de educación media. Folios, 36, 115-130.

Mateos, M. (1999). Metacognición en expertos y novatos. En J. Pozo \& C. Monereo (coord.). El aprendizaje estratégico. Enseñar a aprender desde el currículo (pp. 123-129). Madrid: Santillana.

Mayor, J., Suengas A. \& González, J. (1995). Estrategias metacognitivas. Aprender a aprender y aprender a pensar. Madrid: Síntesis Psicología.

Nieto, D. A. (2005). Efectos del resumen en la mejora de la metacomprensión, de la comprensión lectora y del rendimiento académico. Revista de Educación, 337, 281-294.

Onwuegbuzie, A. J. \& Leech, N. L. (2006, septiembre). Linking Research Questions to Mixed Methods Data Analysis Procedures. Qual Report, 11 (3), 474-498.

Orozco, B. C. (2003). ¿Lee el niño preescolar? En B. C. Orozco (comp.). El niño científico, lector y escritor, matemático (pp. 77-97). Cali: Artes Gráficas del Valle.

Pérez, M. (2003). Leer y escribir en la escuela: algunos escenarios pedagógicos para la reflexión. Bogotá: Icfes.

Perfetti, C. A. (1999). Comprehending written language: a blueprint of the reader. En C. M. Brown y P. Hagoort (eds.). The neurocognition of language (pp. 167-208). Oxford: Oxford University Press.

Pinzas, J. (2003.). Leer mejor para enseñar mejor. Lima: Tarea Asociaciones de Publicaciones Educativas.

Puche-Navarro, R. ; Orozco, M.; Orozco, B. C. \& Correa, M. (2009). Desarrollo infantil y competencias. Bogotá: Ministerio de Educación Nacional, Serie Documentos 10. 
Quaas, C., Ascorra, P. \& Bertoglia, L. (2005). Estrategias metacomprensivas lectoras y constructos asociados: en búsqueda de una relación. Psicoperspectivas, 4 (1), 77-90.

Sánchez, D. L. (10 de septiembre de 2009). Niveles de comprensión lectora. Disponible en: http://letrasuruguay.espaciolatino.com/aaa/sanchez_lihon_ danilo/niveles_de_comprension_lectora.htm

Santibáñez, R. \& Pisconte, M. (2003). Efectos del programa de reforzamiento de lectura orientado al desarrollo de habilidades metalectoras en los alumnos de estudios generales de la Universidad Nacional de Piura. Tesis para optar el grado de magíster en Psicología, Unife, Lima, Perú.

Scarbourough, H. S. (2002). Connecting early language and literacy to later reading (dis)abilities: evidence, theory and practice. En S. B. Neuman \& D. K. Dickinson (eds.). Handbook of Early Literacy Research (pp. 97-110). Nueva York: The Guilford Press.

Schmitt, M. C. \& Baumann, J. F. (1989). Cómo incorporar las estrategias de control de la comprensión a la enseñanza con textos base de lectura. Comunicación, Lenguaje y Educación, 1 (1), 45-50.
Silvestri, A. (2006). La formulación de preguntas para la comprensión de textos: estudio experimental. Revista Signos, 39 (62), 493-510.

Sistema Nacional de Evaluación de la Educación, Icfes (2013). Resultados Icfes Saber $3^{\circ}, 5^{\circ}$ y $9^{\circ} 2012$. Bogotá: autor.

Smith, F. (1985). Reading without nonsense (2nd Edition). Nueva York: Teachers College Press.

Tolchinsky, L. \& Simó, R. (2003). Escribir y leer a través del currículum. Barcelona: Universidad de Barcelona.

Vigotsky, L. V. (1989). El desarrollo de los procesos psicológicos superiores. Barcelona: Crítica.

Wong, M., \& Matalinares, C. (2011). Estrategias de metacomprensión lectora y estilos de aprendizaje en estudiantes universitarios. Revista de Investigación en Psicología, 14 (1), 235-260. 